\title{
The locality of the square-root method for improved staggered quarks
}

\author{
A. Hart $^{1}$ and E. Müller ${ }^{1}$ \\ ${ }^{1}$ School of Physics, University of Edinburgh, King's Buildings, Edinburgh EH9 3JZ, U.K.
}

\begin{abstract}
We study the effects of improvement on the locality of square-rooted staggered Dirac operators in lattice QCD simulations. We find the localisation lengths of the improved operators (FAT7TAD and ASQTAD) to be very similar to that of the one-link operator studied by Bunk et al., being at least the Compton wavelength of the lightest particle in the theory, even in the continuum limit. We conclude that improvement has no effect. We discuss the implications of this result for the locality of the $n^{\text {th }}$-rooted fermion determinant used to reduce the number of sea quark flavours, and for possible staggered valence quark formulations.
\end{abstract}

PACS numbers: 11.15.Ha, 12.38.Gc

\section{INTRODUCTION}

One of the biggest headaches in lattice QCD calculations is the inclusion of quarks in a realistic and readily simulated manner. Staggered fermions have the advantage over Wilson-like quarks that their residual chiral symmetry protects the light quark mass from additive renormalisation. Staggered fermions are also much cheaper to simulate than those from the overlap formulation. The disadvantage is that the formalism naturally yields $N_{f}=4$ degenerate fermion species (known as "tastes"). In the continuum limit these tastes decouple, but at finite lattice spacing, $a$, there are $\mathcal{O}\left(a^{2}\right)$ taste-changing interactions. Improvement of the action can systematically reduce these interactions, leading to smaller splittings in hadron mass multiplets [1], a more physical Dirac spectrum [2, 3] and the correct chiral suppression of the topological susceptibility [4, 5].

True QCD, however, requires a light " $2+1$ "-flavour sea quark mass degeneracy. The usual way of tackling this is to replace the fermion determinant in the partition function by its $n^{\text {th }}$-root, with $n$ either 2 or 4 , to give reduced-taste staggered fermions $[6$, , 7]. This approach is inspired by the continuum factorisation into independent tastes. It is not certain, however, whether this process yields a theory that is sufficiently local that we are confident of the universality in the continuum limit, and will arrive at the correct theory, QCD, as $a \rightarrow 0$.

To answer this we seek to prove at least the existence of a local operator whose determinant matches that of the reduced-taste sea quarks. Explicitly formulating this operator would also provide a consistent description of the valence quarks - currently lacking - and allow an unambiguous perturbative analysis of the theory. Attention so far has focused on the simplest candidate, the $n^{\text {th }}$-rooted Dirac operator, as $(\operatorname{det} \not D)^{1 / n}=\operatorname{det}\left(\not D^{1 / n}\right)$.

The square-root of the simplest, one-link staggered operator was studied in [8]. Free fermions were shown to be non-local both at finite $a$ and in the continuum limit, with a localisation length of order the inverse of the quark mass, $m$. The limits of the eigenvalue spectrum placed a similar upper bound on the interacting theory. Numerical measurements showed the operator had a continuum limit localisation length at least as large as the correlation length of the lightest confined state, the Goldstone pion.

In this Brief Report we investigate to what extent the non-locality (or otherwise) is due to the taste-changing interactions. Using a similar methodology to [8] , we compare the locality of the FAT7TAD and ASQTAD improved staggered operators at various lattice spacings. We show the size of the taste-changing interactions has almost no effect, and all the square-rooted staggered operators are non-local in the continuum limit on a scale at least that of the largest Compton wavelength. We discuss the implications of this for possible reduced-taste formulations for valence and sea quarks in the context of the expected properties of the infrared Dirac spectrum.

\section{METHOD}

An exponential locality length, $r_{\text {loc }}$, can be defined as follows [8]. Given a candidate Dirac operator $\not D(x, y)$, we apply it to a point source $\xi(y)=\delta_{y z}$ to define a wavefunction $\psi(x)=\sum_{y} \not D(x, y) \xi(y)$. The operator is exponentially local if the locality function $f(r)$ is bounded by

$$
f(r) \equiv \max _{\|x-z\|=r}\{|\psi(x)|\} \leqslant \exp \left(-\frac{r}{r_{\text {loc }}}\right) .
$$

We require $r_{\text {loc }} \rightarrow 0$ in the continuum limit to guarantee universality, as has been seen for overlap fermions [9].

We measure Eqn. (11) for the one-link, FAT7TAD and ASQTAD staggered quark formulations [10, 11]. The lattices studied are described in Table 【 We use periodic boundary conditions and study three lattice spacings, choosing the quark mass such that the pion (lightest singlet pseudoscalar meson) mass is fixed in physical units. For compatibility with 8 , we choose $m$ such that $r_{0} m_{\pi} \simeq 1.3($ or $520 \mathrm{MeV})$ 12, 13, 14]. The lattice size is fixed around $1.5 \mathrm{fm}$, or four Compton wavelengths of the pion.

We use the same $m$ for calculations with the improved fermion formulations. Whilst the different renormalisations will change the value of the pion mass, Ref. 10] 
TABLE I: The Wilson gauge action ensembles studied. The $\beta=6.0$ configurations are from [19]. Tadpole improvement factors $u_{0}$ come from the mean plaquette [20]. We set the scale using $r_{0}$ [21].

\begin{tabular}{ccccccc}
\hline \hline$\beta$ & $L^{3} T$ & $N_{\text {conf }}$ & $r_{0}$ & $a L / \mathrm{fm}$ & $u_{0}$ & $m$ \\
\hline 5.8 & $12^{4}$ & 107 & $3.673(5)$ & 1.63 & 0.8680 & 0.017 \\
6.0 & $16^{3} 32$ & 221 & $5.371(15)$ & 1.49 & 0.8778 & 0.010 \\
6.2 & $24^{4}$ & 94 & $7.380(26)$ & 1.63 & 0.8851 & 0.007 \\
\hline \hline
\end{tabular}

shows this effect is only slight, and does not affect the conclusions of this study.

If $M$ is the staggered Dirac operator, then $M^{\dagger} M$ defined on the even sites is an Hermitian, positive-definite matrix representing $N_{f}=4$ tastes [15, 16]. We study the locality of $\not D=\sqrt{M^{\dagger} M}$ using Eqn. (11). In this, $|\cdot|$ is the $\mathrm{SU}(3)$ colour norm, and $\|\cdot\|$ the periodic $L_{1}$ ("taxi driver") norm [8].

The square-root function is approximated using a Tchebyshev polynomial [17] of order $n_{\text {poly }}=500$. The analytic upper bound on the truncation error is $\mathcal{O}\left(10^{-2}\right)$ [18]. Before discussing the results, we must first be sure that the truncation errors in the polynomial do not affect our estimates of the locality function.

Measuring $f(r)$ for various $n_{\text {poly }} \leqslant 500$, the error should decrease exponentially, and so

$$
\begin{aligned}
\Delta\left(r, n_{\text {poly }}\right) & \equiv\left|f\left(r, n_{\text {poly }}\right)-f(r, 500)\right| \\
& =A e^{-B n_{\text {poly }}}\left(1-e^{-B\left(500-n_{\text {poly }}\right)}\right) \\
& \simeq A e^{-B n_{\text {poly }}} \text { for } 0<n_{\text {poly }} \ll 500
\end{aligned}
$$

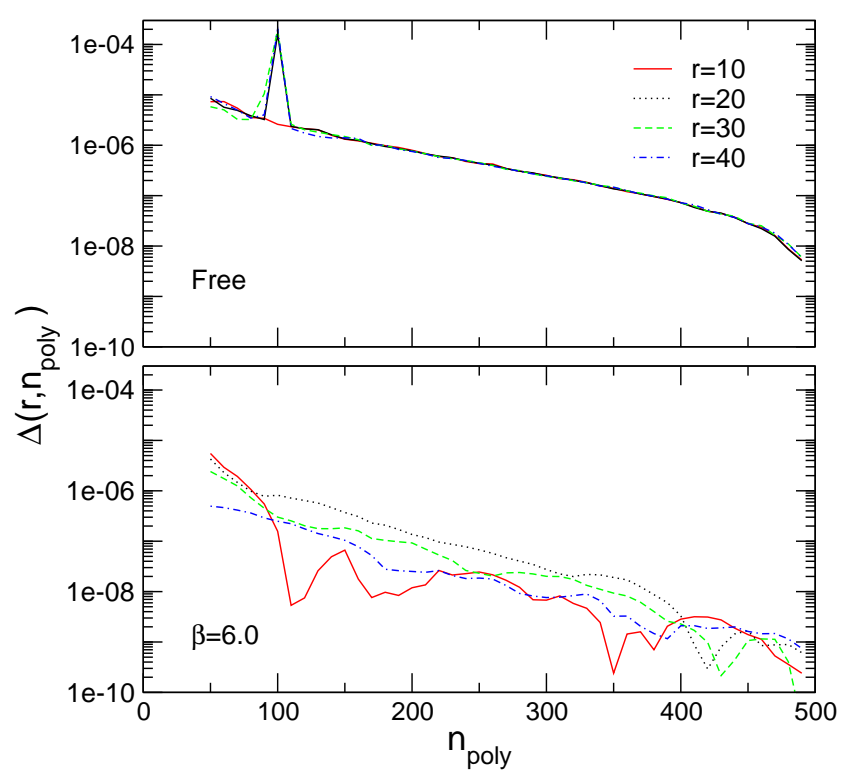

FIG. 1: Relative accuracy of the localisation function using the polynomial approximation to the square root of the onelink staggered action. Measurements are on $16^{3} 32$ for the free theory and $\beta=6.0$.

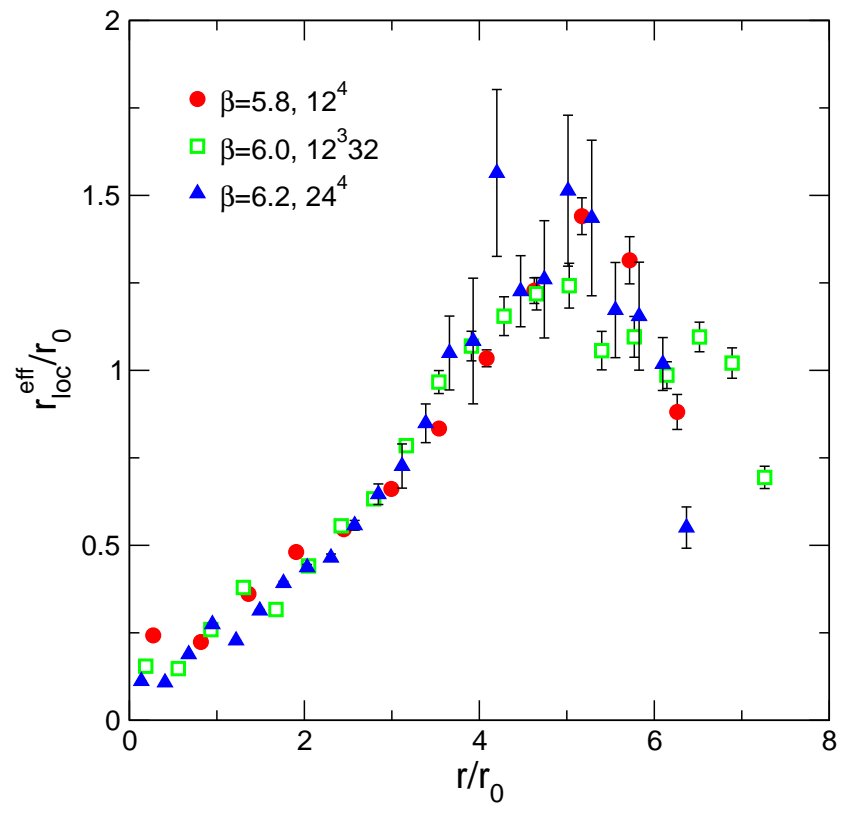

FIG. 2: Scaling of the effective localisation length for reduced-flavour $N_{f}=2$ ASQTAD fermions.

and $A(r), B(r)>0$. We plot $\Delta$ at various fixed $r$ in Fig. 1] for free fermions and for a representative gauge background at $\beta=6.0$. Extrapolating the linear region to $n_{\text {poly }}=500$, we estimate the truncation error to be $\mathcal{O}\left(10^{-8}\right)$. This is an order of magnitude smaller than the minimum of $f(r)$. As improved fermions should lie somewhere between the free and the one-link interacting case, we use $n_{\text {poly }}=500$ in all our analysis.

We may ask why the actual error is so much smaller than the theoretical bound. A heuristic argument is that the errors on the Tchebyshev approximation are heavily concentrated at the lower end of the spectrum. The eigenvalues of the Dirac operator are, by contrast, relatively sparse here. Assuming the localisation function is not completely dominated by the infrared modes, it is reasonable that truncation errors towards the middle of the operator spectrum (which are many of orders of magnitude smaller) are more representative of the error on $f(r)$.

\section{RESULTS}

Using the locality function, we define an effective localisation length $r_{\text {loc }}^{\text {eff }}(r)$ at distance $r$ by

$$
r_{\mathrm{loc}}^{\mathrm{eff}}\left(\frac{r_{i}+r_{i+1}}{2}\right)=\frac{\left(r_{i+1}-r_{i}\right)}{\log \left(f\left(r_{i}\right) / f\left(r_{i+1}\right)\right)}
$$

for each pair of subsequent distances $r_{i}$.

We plot this function for the square-rooted ASQTAD operator in Fig. 2 scaling everything in units of $r_{0}$. At small distances $f(r)$ is almost a power law (with exponent somewhere between -3 and -4 ). Comparing our 


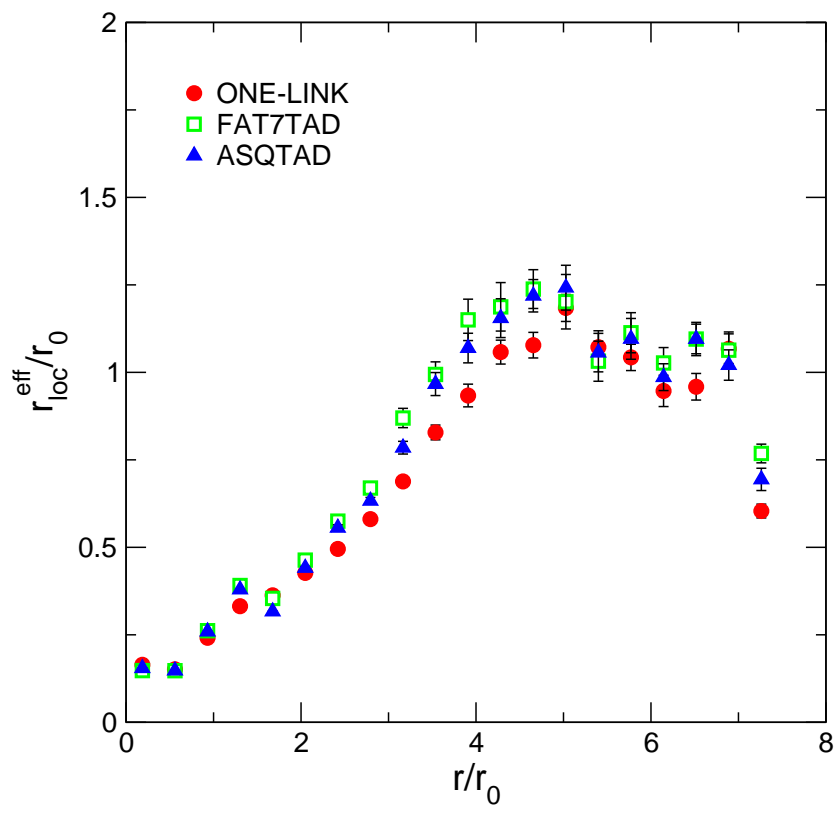

FIG. 3: A comparison of the effective locality length for various reduced-flavour $N_{f}=2$ staggered operators at $\beta=$ 6.0 .

results with larger volumes $a L \simeq 2.1 \mathrm{fm}$, only results for $r / r_{0} \lesssim 3$ are free of finite volume effects. In this range there is no clear evidence for a crossover to exponential locality, so localisation length estimates must be treated as lower bounds only. Nonetheless, these bounds do not scale to zero with the lattice spacing, but rather remain constant. It therefore seems highly likely that the actual localisation length also remains finite in physical units in the continuum limit.

We conclude, then, that the square-rooted ASQTAD Dirac operator is severely non-local in the continuum limit. In Fig. 3 we compare at fixed lattice spacing the three operators. The effective locality lengths are extremely similar. This was also the case at the other lattice spacings. It would therefore appear that the improvement programme for staggered quarks has no effect on the locality of the square-rooted Dirac operator.

\section{DISCUSSION}

Understanding how to consistently reduce the number of staggered tastes for the sea and valence quarks is vital to realistic QCD simulations. A proposed solution for the sea quarks is to take the $n^{\text {th }}$-root of the fermion determinant. The locality (and legitimacy) of the reduced-taste theory is not clear, but may be answered by attempting to find a local reduced-taste Dirac operator with the same determinant. Using this same operator to describe the valence quarks (rather than the $N_{f}=4$ staggered operator used at present) would also avoid the poten- tial mode mismatches seen for other mixed valence/sea fermion combinations 22 .

We have examined a candidate two-flavour Dirac operator, inspired by the $n^{\text {th }}$-root trick used to reduce the number of sea quark tastes. We have seen that the locality of this square-rooted operator is as bad for improved staggered formulations (FAT7TAD and ASQTAD) as it was for the one-link case [8]: the locality length for $5.8 \leqslant \beta \leqslant 6.2$ shows no signs that $r_{\text {loc }}$ vanishes in the continuum limit. By contrast, $r_{\text {loc }}$ appears to be at least the Compton wavelength of the lightest particle.

In many areas improved staggered quarks show a definite improvement over the one-link case $1,[2,4,[5]$. That there is almost no commensurate change in the locality suggests that the $n^{\text {th }}$-rooted operator is not a useful way of formulating the valence quarks. It is therefore unlikely to be a good choice for studying the locality of the determinant; a better choice would be an operator with more physical properties.

Given a sufficiently smooth gauge background of topological charge $Q$, a lattice Dirac operator should have an infrared spectrum characteristic of the number of tastes/flavours it represents. The Atiyah-Singer Index Theorem predicts that there should be (at least) $N_{f}|Q|$ near-zero modes 2, 3, 23]. The remaining low-lying modes should lie in near-degenerate $N_{f}$-plets, with any splitting due to taste-changing interactions [2, 3]. The multiplets should follow a universal distribution given, for instance, by random matrix theory [2, 24]. Taking the $n^{\text {th }}$-root will reduce neither the number of zero modes nor the multiplicity of other eigenvalues by factors of $n$. In addition, eigenvalue ratios $\left\langle\lambda_{s}^{1 / n}\right\rangle /\left\langle\lambda_{t}^{1 / n}\right\rangle$ can no longer agree with the universal distribution.

By analogy with the reduction of 16 naivve fermion species to 4 ultra-local staggered tastes, it seems likely that a physical reduced-taste staggered Dirac operator will, if it exists, be obtained by projection rather than rooting. Unlike naïve to staggered, however, tastebreaking interactions prevent this being exact at finite lattice spacing, but improved staggered spectra already show much closer taste symmetry [2, 3]. It is not clear whether such a reduced-taste operator would be local or even renormalisable. It is, however, important to study this to understand the systematic uncertainty of using the $n^{\text {th }}$-root method in precision lattice QCD simulations.

\section{Acknowledgments}

We are grateful to K. Jansen and A.D. Kennedy for useful discussions, as well as A.D.K. for a critical reading of the manuscript. A.H. thanks the U.K. Royal Society, and E.M. the DAAD German Academic Exchange Service for financial support. 
[1] E. Follana et al., Nucl. Phys. Proc. Suppl. 129, 384 (2004), [hep-lat/0406021].

[2] E. Follana, A. Hart and C. T. H. Davies, heplat/0406010.

[3] S. Dürr, C. Hoelbling and U. Wenger, hep-lat/0406027.

[4] MILC, C. Bernard et al., Phys. Rev. D68, 114501 (2003), [hep-lat/0308019].

[5] A. Hasenfratz, Phys. Rev. D64, 074503 (2001), [heplat/0104015].

[6] F. Fucito, E. Marinari, G. Parisi and C. Rebbi, Nucl. Phys. B180, 369 (1981).

[7] E. Marinari, G. Parisi and C. Rebbi, Nucl. Phys. B190, 734 (1981).

[8] B. Bunk, M. Della Morte, K. Jansen and F. Knechtli, hep-lat/0403022.

[9] P. Hernandez, K. Jansen and M. Luscher, Nucl. Phys. B552, 363 (1999), [hep-lat/9808010].

[10] MILC, K. Orginos and D. Toussaint, Phys. Rev. D59, 014501 (1999), [hep-lat/9805009].

[11] MILC, K. Orginos, D. Toussaint and R. L. Sugar, Phys. Rev. D60, 054503 (1999), [hep-lat/9903032].

[12] R. Gupta, G. Guralnik, G. W. Kilcup and S. R. Sharpe, Phys. Rev. D43, 2003 (1991).

[13] S. Kim and D. K. Sinclair, Phys. Rev. D52, 2614 (1995), [hep-lat/9502004].

[14] S. Kim and S. Ohta, Phys. Rev. D61, 074506 (2000), [hep-lat/9912001].

[15] O. Martin and S. W. Otto, Phys. Rev. D31, 435 (1985).

[16] J. Polonyi, H. W. Wyld, J. B. Kogut, J. Shigemitsu and D. K. Sinclair, Phys. Rev. Lett. 53, 644 (1984).

[17] W. Press et al., Numerical Recipes: the Art of Scientific Computing, 2nd ed. (CUP, 1992).

[18] A. D. Kennedy, Nucl. Phys. Proc. Suppl. 128C, 107 (2004), [hep-lat/0402037].

[19] G. Kilcup, D. Pekurovsky and L. Venkataraman, Nucl. Phys. Proc. Suppl. 53, 345 (1997), [hep-lat/9609006], available from the NERSC Gauge Connection archive http://qcd.nersc.gov.

[20] B. Lucini and M. Teper, JHEP 06, 050 (2001), [heplat/0103027].

[21] S. Necco and R. Sommer, Nucl. Phys. B622, 328 (2002), [hep-lat/0108008].

[22] S. Dürr and C. Hoelbling, Phys. Rev. D69, 034503 (2004), [hep-lat/0311002].

[23] J. Smit and J. C. Vink, Nucl. Phys. B286, 485 (1987).

[24] E. V. Shuryak and J. J. M. Verbaarschot, Nucl. Phys. A560, 306 (1993), [hep-th/9212088]. 